\title{
Cerebrospinal Fluid Corticotropin-Releasing Factor Concentration is Associated with Pain but not Fatigue Symptoms in Patients with Fibromyalgia
}

\begin{abstract}
Samuel A McLean*, , David A Williams², Phyllis K Stein ${ }^{3}$, Richard E Harris², Angela K Lyden², Gail Whalen ${ }^{4}$, Karen M Park ${ }^{5}$, Israel Liberzon ${ }^{6}$, Ananda Sen ${ }^{7}$, Richard H Gracely, James N Baraniuk ${ }^{4}$ and Daniel J Clauw ${ }^{2}$

'Department of Emergency Medicine, University of Michigan Medical Center, Ann Arbor, MI, USA; '2Department of Medicine (Rheumatology), University of Michigan Medical Center, Ann Arbor, MI, USA; ${ }^{3}$ Heart Rate Variability Laboratory, Cardiovascular Division, Washington University School of Medicine, St Louis, MO, USA; ${ }^{4}$ Department of Medicine (Rheumatology), Georgetown University Medical Center, Washington, DC, USA; ${ }^{5}$ Michigan Pain Specialists, Ann Arbor, MI, USA; ${ }^{6}$ Department of Psychiatry, University of Michigan Medical Center, Ann Arbor, MI, USA; ${ }^{7}$ Center for Statistical Consulting and Research, University of Michigan Medical Center, Ann Arbor, MI, USA
\end{abstract}

Previous studies have identified stress system dysregulation in fibromyalgia (FM) patients; such dysregulation may be involved in the generation and/or maintenance of pain and other symptoms. Corticotropin-releasing factor (CRF) is the principal known central nervous system mediator of the stress response; however, to date no studies have examined cerebrospinal fluid (CSF) CRF levels in patients with FM. The relationship between CSF CRF level, heart rate variability (HRV), and pain, fatigue, and depressive symptoms was examined in patients with FM. Among participants $(n=26)$, CSF CRF levels were associated with sensory pain symptoms $(r=0.574, p=0.003)$ and affective pain symptoms $(r=0.497, p=0.0 \mathrm{I})$ ), but not fatigue symptoms. Increased HRV was also strongly associated with increased CSF CRF and FM pain. In multivariate analyses adjusting for age, sex, and depressive symptoms, the association between CSF CRF and sensory pain symptoms ( $t=2.54, p=0.027$ ) persisted. Women with FM who reported a history of physical or sexual abuse had lower CSF CRF levels than women who did not report such a history. CSF CRF levels are associated with both pain symptoms and variation in autonomic function in FM. Differences in CSF CRF levels among women with and without a self-reported history of physical or sexual abuse suggest that subgroups of FM patients may exist with different neurobiological characteristics. Further studies are needed to better understand the nature of the association between CSF CRF and pain symptoms in FM.

Neuropsychopharmacology (2006) 3 I, 2776-2782. doi: I0.I038/sj.npp. I30 I200; published online 23 August 2006

Keywords: fibromyalgia; corticotrophin-releasing factor; depression; abuse; heart rate variability

\section{INTRODUCTION}

Fibromyalgia (FM) is a common clinical syndrome defined by the presence of chronic widespread pain and increased tenderness to palpation (Wolfe et al, 1990). Recent studies have identified altered central nervous system pain processing in individuals with FM, supporting a neurobiological basis for the disorder (Gracely et al, 2002; Cook et al, 2004). However, the specific pathophysiologic mechanisms responsible for FM remain poorly understood.

Scientific Meeting Presentation: American College of Rheumatology, San Antonio, Texas, October 2004

*Correspondence: Dr SA McLean, University of Michigan Chronic Pain and Fatigue Research Center, 24 Frank Lloyd Wright Drive, PO Box 385, Ann Arbor, MI 48I06, USA, Tel: + I 734615 2765, Fax: + I 734 998 6900, E-mail: samclean@umich.edu

Received 10 March 2006; revised 7 June 2006; accepted 25 July 2006 Online publication: 27 July 2006 at http://www.acnp.org/citations/ Npp072706060 162/default.pdf
Some authors have proposed that disruptions in stress system function may be an important mechanism generating pain and other symptoms in FM (Chrousos and Gold, 1992; Crofford and Demitrack, 1996; Clauw and Chrousos, 1997). The principal components of the human stress response are the hypothalamic-pituitary-adrenocortical (HPA) axis and locus ceruleus/norepinephrine-sympathetic (LC-NE) system (Chrousos and Gold, 1992). Results of studies examining HPA axis function in patients with FM are inconsistent, with both HPA axis hypoactivity and hyperactivity identified (McCain and Tilbe, 1989; Griep et al, 1993; Crofford et al, 1994; Riedel et al, 1998; Catley et al, 2000). Autonomic nervous system function in patients with FM, reflected in both heart rate variability (HRV) and plasma catecholamine levels, suggests a central hypernoradrenergic state (Martinez-Lavin et al, 1998; Stein et al, 2004).

Corticotropin-releasing factor (CRF) is the principal known central nervous system mediator of the pituitary- 
adrenal response to stress. CRF appears to be important to the pathophysiology of stress-related disorders such as post-traumatic stress disorder (PTSD) and depression; elevated levels of cerebrospinal fluid (CSF) CRF have been identified in both of these disorders (Bremner et al, 1997; Gold and Chrousos, 2002). Early life stress has also been associated with alterations in adult CSF CRF levels, in both preclinical and clinical studies (Carpenter et al, 2004; Heim et al, 2004), and recent study results indicate that that early life stress influences stress system function in adults with FM (McLean et al, 2005b; Weissbecker et al, 2005). In the single study assessing CRF levels in FM patients to date, Riedel et al (2002) found higher baseline serum CRF levels in FM patients $v s$ healthy controls. To date, we are not aware of any studies that have examined CSF CRF concentrations among patients with FM.

In this study, we sought to identify the relationship between CSF CRF concentration and FM symptoms (ie pain, fatigue, and depressive symptoms), and between CSF CRF and measures of autonomic nervous system function (ie HRV) among patients with FM. We hypothesized that CSF CRF levels would be positively associated with pain and fatigue symptoms. We also hypothesized that increased CSF CRF levels would be associated with increased sympathetic nervous system activity among FM patients, given the identified association between CSF CRF and the LE-NE system (Palkovits, 1999). In addition, among women with FM, we explored the relationship between self-reported physical or sexual abuse and CSF CRF level.

\section{PATIENTS AND METHODS}

\section{Patients}

The study sample consisted of participants with FM $(n=26)$ recruited via local print advertisements and clinic samples. All participants underwent a detailed evaluation to exclude other medical conditions, including history and physical examination, and laboratory studies including $\mathrm{CBC}$, serum electrolytes, BUN, Cr, TSH, sedimentation rate, and C-reactive protein. General exclusion criteria were as follows: (1) cigarette smoking; (2) substance abuse in the past 2 years; (3) medical conditions known to cause symptoms similar to FM symptomatology, including obesity $\left(\mathrm{BMI}>30 \mathrm{~kg} / \mathrm{m}^{2}\right)$, autoimmune or inflammatory diseases, cardiopulmonary disorders, chronic asthma, uncontrolled endocrine, or allergic disorders (eg hypothyroidism, diabetes, allergic rhinitis), or malignancy; or (3) schizophrenia or major depression with suicidal ideation.

All participants were discontinued from psychoactive medications prior to study onset. Fluoxetine taper was completed at least 4 weeks prior to study initiation (two patients); other psychoactive medications were tapered off at least 2 weeks prior to study initiation. These other medications included other antidepressants (paroxetine (four patients), bupropion (one patient), trazodone (three patients), sertraline (two patients), nortriptyline (one patient), and amitriptyline (one patient)), other pain medications (methocarbamol (one patient), cyclobenzaprine (three patients), gabapentin (one patient), lidocaine patch (one patient), methadone (one patient), and oxycodone (one patient)), and benzodiazepines (lorazepam (one patient), diazepam (one patient), clonazepam (one patient), and zolpidem (one patient)). Nonsteroidal anti-inflammatory drugs were discontinued at least 3 days prior to the study (nine patients).

All participants received specialized evaluation to determine if they met the 1990 American College of Rheumatology criteria for the classification of FM (Wolfe et al, 1990). Patients who reported a history of FM but did not meet ACR criteria at the time of the study were excluded. The presence of psychiatric disorders was assessed using the Composite International Diagnostic Interview (Wittchen, 1994). In addition, participants were asked if they had ever been the victim of physical or sexual abuse. No specific definition of physical or sexual abuse was provided. If a participant did report abuse, they were asked the age at which the physical or sexual abuse occurred or began.

\section{Self-Report Instruments}

Participants completed the following self-report instruments, each of which was chosen based on its psychometric properties and applicability to the FM population:

Short-Form of the McGill Pain Questionnaire: This SFMPQ questionnaire has been extensively evaluated and contains 15 pain adjectives (Melzack, 1987). A sensory score is obtained by summing 11 of the items, an affective score is obtained by summing the remaining four items, and a total score is obtained by summing all of the items.

The Multidimensional Fatigue Inventory (MFI): The MFI is a 20 -item instrument that assesses the following dimensions of fatigue: general fatigue, physical fatigue, mental fatigue, reduced activity, and reduced motivation. It has been validated among multiple patient populations, including individuals with chronic fatigue syndrome (CFS) (Smets et al, 1995).

The Center for Epidemiological Studies Depression Scale (CES-D): The CES-D is a 20-item measure that assesses multiple components of depressive symptomatology: depressed mood, feelings of guilt and worthlessness, feelings of helplessness and hopelessness, loss of appetite, sleep disturbance, and psychomotor retardation. The CES-D has demonstrated strong associations with other depressive symptom measures (Radloff, 1977) and has been validated in pain populations (Geisser et al, 1997).

\section{Procedures}

The study protocol included a 2-day in-patient evaluation conducted in the Georgetown University Medical Center's General Clinical Research Center (GCRC). Menstruating women were scheduled to undergo study evaluations during days 3-7 of the follicular phase of their menstrual cycle. The in-patient GCRC evaluation began at 1800 hours. Participants initially completed self-report questionnaires, then had a Holter monitor (ACS Holter, Ontario, CA) placed at 2000 hours. This Holter monitor was worn overnight and during day 1 of the GCRC protocol. This protocol included several participant stressors, including evoked pain testing, cognitive testing, and exercise testing via cycle ergometer. The Holter monitor was removed at 1700 hours, after completion of the day's protocol. Participants then spent another night in the GCRC, followed by a lumbar puncture 
performed at 0830 hours the next morning by a boardcertified anesthesiologist. Participants had nothing to eat or drink after midnight prior to the test. The study protocol was approved by the Georgetown University Institutional Review Board, and all participants gave informed consent prior to protocol enrollment. Participants were compensated $\$ 425$.

Holter monitor data was used to assess autonomic nervous system function via HRV. Autonomic dysfunction has been identified in patients with FM (Radulovic et al, 1995), and multiple lines of evidence suggest an association between CRF levels and LC-NE/autonomic nervous system function (Gold and Chrousos, 2002). HRV represents the beat-to-beat variation in consecutive $R-R$ intervals. Fluctuations over time in these intervals are mediated by autonomic inputs to the sinus node, and measures of HRV represent a surrogate measure of autonomic nervous system modulation. HRV was assessed across the different domains of the heart rate power spectrum (frequency domain analysis), including power in the ultra-low- (ULF), very low- (VLF), low- (LF), and high-frequency (HF) bands. The LF to HF power ratio was also calculated. Power in the HF band $(0.15-0.4 \mathrm{~Hz})$ largely reflects respiration-mediated vagal contributions to the beat-to-beat variations in heart rate (Stein and Kleiger, 1999). The LF band is thought to be modulated by both the sympathetic and parasympathetic nervous systems. It is measured between 0.04 and $0.15 \mathrm{~Hz}$ (Stein and Kleiger, 1999). Even slower modulations of heart rate are reflected in the VLF domain $(0.0033-0.04 \mathrm{~Hz})$. VLF was long believed to represent the influence of the peripheral vasomotor and reninangiotensin systems (Akselrod et al, 1981); however, recent studies using pharmacological blockade support a strong contribution of the parasympathetic nervous system to VLF (Taylor et al, 1998). The remainder of the power spectrum is subsumed by the ULF domain, which reflects all variance below $0.0033 \mathrm{~Hz}$, and consists of mainly circadian rhythms. All of these indices together represent the total power, which is the sum of all the variance in the heart period signal. The LF/HF ratio is a rough estimate of fluctuations in the balance between the sympathetic and parasympathetic nervous systems (Stein and Kleiger, 1999).

Three samples of CSF, $3 \mathrm{~cm}^{3}$ each, were obtained via lumbar puncture. The third sample was immediately spun, aliquoted, and stored at $-70^{\circ} \mathrm{C}$ for later batch analysis of CSF CRF. Concentrations of CSF CRF were measured using radioimmunoassay (Peninsula Laboratories, San Carlos, $\mathrm{CA}$; detection limit, $1 \mathrm{pg} / 100 \mu \mathrm{l})$.

\section{Statistical Analysis}

All study data were entered onto a secure database via double data entry. CSF CRF levels among male and female participants were compared via the Wilcoxon-MannWhitney test. Partial correlations between CSF CRF and demographic, history, and symptom characteristics and autonomic nervous system function were calculated using Spearman's rank correlation, adjusting for age and sex. Multiple linear regression analysis was used to assess the amount of variance in CSF CRF explained by demographic and symptom characteristics and autonomic nervous system function, and to assess the association between pain symptoms and CSF CRF, adjusted for age, sex, and depressive symptoms. In addition, among women with FM, the association between self-reported abuse history and CSF CRF was assessed, as well as the amount of variance in CSF CRF explained by abuse history, depressive symptoms, and McGill sensory subscale score.

Possible interactions were assessed in preliminary models. If no significant interaction was present, the interaction term was excluded from the final model. The variance in the dependent variable explained by the regression model was assessed via adjusted $R^{2}$. Goodness of fit and model aptness were evaluated via residual analysis. All regression models were evaluated for normality and other diagnostic issues. For Tables 4 and 5, the McGill pain scales and CSF CRF significantly deviated from normality and were symmetrized by using a natural log transformation. Statistical analyses were performed using SPSS software (SPSS Inc., Chicago, IL).

\section{RESULTS}

\section{Participant Characteristics}

Participant demographic and symptom characteristics and CSF CRF levels are shown in Table 1. CSF CRF levels were higher in males than in females $(p=0.03)$. Consistent with prior reports (Clauw and Chrousos, 1997), CFS defined via the CDC criteria (Fukuda et al, 1994) often coincided with FM (22 of 26 patients; $85 \%$ ).

\section{CSF CRF Levels and FM Symptom Characteristics and Autonomic Nervous System Function}

Table 2 presents the partial correlations between CSF CRF and FM symptom characteristics and autonomic nervous system function, adjusted for age and sex. Increased CSF CRF was strongly associated with increased pain symptoms, but not fatigue symptoms. CSF CRF was also strongly associated with autonomic nervous system function, particularly the LF portion of the spectrum, which is consistent with increased sympathetic activity. Sixty-six percent of the variation in CSF CRF was accounted for by a regression model containing McGill pain sensory score, LF HRV, age, gender, and depressive symptoms (Table 3).

To further examine the relationship between CSF CRF and patient pain and depressive symptoms, multiple linear regression analyses were performed using McGill sensory and affective subscale scores as dependent variables (Table 4). The strong association between McGill sensory and affective subscale scores and CSF CRF persisted after adjustment for depressive symptoms. These two models accounted for 45 and 24\% of the variation in McGill sensory and affective subscale scores, respectively.

\section{Self-Reported History of Physical or Sexual Abuse and CSF CRF among Women with FM}

During the initial screening examination, participants were asked if they had ever been physically or sexually abused, and, if so, the age at which any such abuse occurred or 
Table I Characteristics of Study Participants $(n=26)$

\begin{tabular}{|c|c|}
\hline Characteristic & Mean (SD) or $N(\%)$ \\
\hline Age (mean, SD) & $43(9)$ \\
\hline $\mathrm{BMI}($ mean, SD) & $26.4(4)$ \\
\hline Male (n, \%) & $10(38)$ \\
\hline \multicolumn{2}{|l|}{ Race (n, \%) } \\
\hline White & $13(52)$ \\
\hline Black & $7(28)$ \\
\hline Hispanic & $3(12)$ \\
\hline Other & $3(12)$ \\
\hline \multicolumn{2}{|l|}{ Symptom score (mean, SD) } \\
\hline McGill sensory & $10(6)$ \\
\hline McGill affective & $3(2)$ \\
\hline $\mathrm{MFI}$ _general fatigue & $16.2(2)$ \\
\hline MFI_mental fatigue & $14.2(4)$ \\
\hline MFI_-physical fatigue & $14.2(4)$ \\
\hline MFI_reduced activity & $11.8(5)$ \\
\hline MFI_reduced motivation & $10.5(4)$ \\
\hline CES-D & $16.4(9)$ \\
\hline \multicolumn{2}{|l|}{ Education $(n, \%)^{\mathrm{a}}$} \\
\hline High school & | (4\%) \\
\hline Some college & II (42\%) \\
\hline College graduate & $9(35 \%)$ \\
\hline Postgraduate degree & $4(15 \%)$ \\
\hline \multicolumn{2}{|l|}{ CSF CRF level (mean, SD (pg/ml)) } \\
\hline All participants $(n=26)$ & $31.8(12.5)$ \\
\hline FM men $(n=10)$ & $38.9(\mid 4.0)$ \\
\hline FM women reporting abuse $(n=7)$ & $25.4(11.5)$ \\
\hline FM women not reporting abuse $(n=9)$ & $35.0(12.4)$ \\
\hline
\end{tabular}

BMI, body mass index; MFI, multidimensional fatigue inventory; CES-D, Center for Epidemiologic Studies-Depression Scale.

aEducation information missing on one participant.

began. Seven of the 16 women with FM reported an abuse history. Five of these women stated that the abuse began between 5 and 13 years of age, and the other two women reported abuse as adults. Two of the women who reported abuse during their preteen years met CIDI criteria for PTSD. Among women with FM in our sample, abuse history was not correlated with number of depressive symptoms $(r=0.14, p=0.66)$. As shown in Figure 1, women reporting a history of physical or sexual abuse had a significantly lower CSF CRF than women not reporting such a history (22 $v s 32 \mathrm{pg} / \mathrm{ml}, P=0.012)$. This group difference persisted when the FM woman not reporting abuse with the highest CSF CRF value $(50 \mathrm{pg} / \mathrm{ml})$ was removed $(p=0.020)$. Among women with $\mathrm{FM}, 64 \%$ of the variance in CSF CRF was accounted for by a model that contained McGill pain sensory score, self-reported abuse history, and depressive symptoms (Table 5).
Table 2 Partial Correlation between CSF CRF and Fibromyalgia Symptom Characteristics and Autonomic Nervous System Function, Adjusted for Age and Sex

\begin{tabular}{lll}
\hline Characteristic & $\boldsymbol{r}$ & p-value \\
\hline Symptom score & & \\
McGill sensory & 0.574 & 0.003 \\
McGill affective & 0.497 & 0.011 \\
MFI_-general fatigue & 0.163 & 0.436 \\
MFI_-mental fatigue & 0.067 & 0.752 \\
MFI_physical fatigue & 0.322 & 0.117 \\
MFI_reduced activity & 0.327 & 0.111 \\
MFI_reduced motivation & 0.346 & 0.090 \\
CES-D (SD) & 0.458 & 0.021 \\
& & \\
Autonomic measures & & 0.028 \\
TP (ms $\left.{ }^{2}\right)$ & 0.497 & 0.012 \\
HF $\left(\mathrm{ms}^{2}\right)$ & 0.579 & 0.003 \\
LF $\left(\mathrm{ms}^{2}\right)$ & 0.656 & 0.057 \\
VLF $\left(\mathrm{ms}^{2}\right)$ & 0.456 & 0.305 \\
LF/HF ratio & -0.256 & \\
\hline CSF CRF, cer & &
\end{tabular}

CSF CRF, cerebrospinal fluid corticotrophin-releasing factor; MFI,

multidimensional fatigue inventory; CES-D, Center for Epidemiologic StudiesDepression Scale; TP, total power heart rate variability; HF, high-frequency heart rate variability; LF, low-frequency heart rate variability; VLF, very-low-frequency heart rate variability.

Table 3 Association between CSF CRF and Patient Characteristics and Autonomic Nervous System Function

\begin{tabular}{llccc}
\hline $\begin{array}{l}\text { Dependent } \\
\text { measure }\end{array}$ & $\begin{array}{l}\text { Independent } \\
\text { variable }\end{array}$ & Beta & $\boldsymbol{t}$ & $\boldsymbol{p}$-value \\
\hline CSF CRF & Age & 0.359 & 2.034 & 0.067 \\
& Sex & 0.387 & 1.955 & 0.076 \\
& McGill sensory & 0.456 & 2.545 & 0.027 \\
& CES-D & 0.278 & 1.796 & 0.100 \\
& LF $\left(\mathrm{ms}^{2}\right)$ & 0.555 & 3.049 & 0.011 \\
\hline
\end{tabular}

CSF CRF, cerebrospinal fluid corticotrophin-releasing factor; CES-D, Center for Epidemiologic Studies-Depression Scale; LF, low-frequency heart rate variability.

Table 4 Association between Patient Pain Symptoms and CSF CRF, Adjusted for Age, Sex, and Depressive symptoms

\begin{tabular}{llrrr}
\hline $\begin{array}{l}\text { Dependent } \\
\text { measure }\end{array}$ & $\begin{array}{l}\text { Independent } \\
\text { variables }\end{array}$ & Beta & t & p-value \\
\hline McGill sensory & Age & -0.189 & -1.138 & 0.268 \\
(Natural Log) & Sex & -0.686 & -3.604 & 0.002 \\
& CES-D & -0.003 & -0.014 & 0.989 \\
& CSF CRF & 0.559 & 2.876 & 0.009 \\
McGill affective & & & & \\
(Natural Log) & Age & -0.302 & -1.629 & 0.119 \\
& Sex & -0.382 & -1.794 & 0.088 \\
& CES-D & 0.048 & 0.246 & 0.808 \\
& CSF CRF & 0.557 & 2.559 & 0.019 \\
\hline
\end{tabular}

CES-D, Center for Epidemiologic Studies-Depression Scale; CSF CRF, cerebrospinal fluid corticotrophin-releasing factor. 


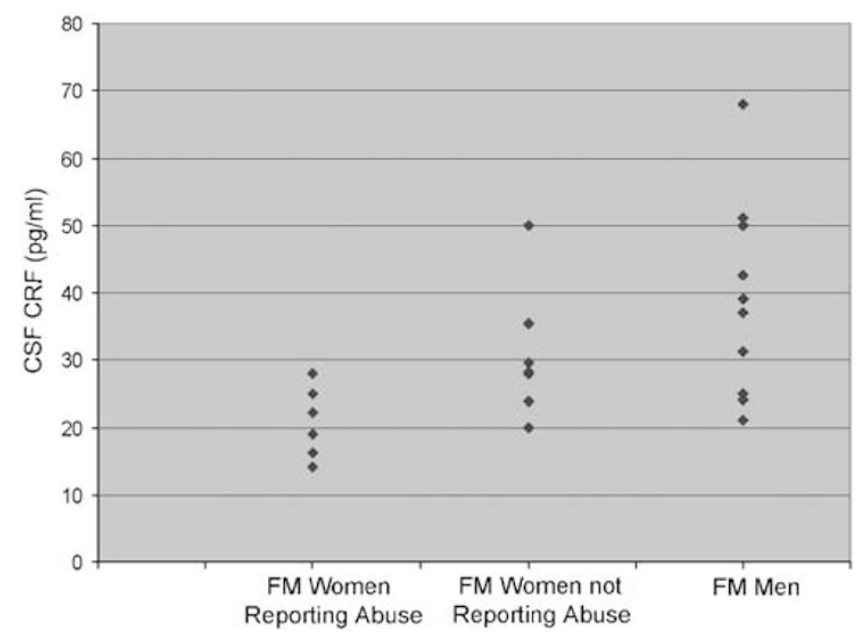

Figure I CSF CRF level among FM women reporting and not reporting a history of abuse, and among men with FM.

Table 5 Association between CSF CRF and Self-Reported Abuse History, Depressive Symptoms, and Pain Symptoms among Women with Fibromyalgia

\begin{tabular}{lllll}
\hline $\begin{array}{l}\text { Dependent } \\
\text { measure }\end{array}$ & $\begin{array}{l}\text { Independent } \\
\text { variable }\end{array}$ & Beta & t & p-value \\
\hline CSF CRF & Abuse history & 0.392 & 3.26 & 0.009 \\
(Natural Log) & CES-D & 0.016 & 2.48 & 0.033 \\
& McGill sensory & 0.019 & 1.898 & 0.087
\end{tabular}

CSF CRF, cerebrospinal fluid corticotrophin-releasing factor; CES-D, Center for Epidemiologic Studies-Depression Scale.

\section{DISCUSSION}

Among patients with FM, pain symptoms, but not fatigue symptoms, were strongly associated with CSF CRF concentration. This association was not accounted for by differences in age, sex, or depressive symptoms. FM pain symptoms were consistently more strongly associated with CSF CRF than were depressive symptoms. Increased HRV, in a manner consistent with elevated sympathetic nervous system activity, was also associated with increased CSF CRF and with pain symptoms. This association is consistent with the interconnected function of the CRF and LC-NE systems (Arlt et al, 2003).

There are three possible explanations for the association between CSF CRF and pain symptoms in patients with FM: CRF may be altered as a consequence of chronic stress caused by pain, CRF may be involved in the generation of pain symptoms, and/or CRF may be a 'third variable' that is altered because of the dysfunction of other central processes directly involved in the generation of FM pain. Numerous animal studies have demonstrated that CRF is capable of influencing nociception (Lariviere and Melzack, 2000), but there is little direct evidence to distinguish between these alternatives. Indirect evidence that stress system dysregulation (due to genetic or environmental influences) is involved in the pathogenesis of FM (McLean et al, 2005a) suggests that CRF levels may be altered by mechanisms other than the chronic stress of illness alone. The results of this study, combined with the findings in a recent study that elevated cortisol levels are associated with FM pain but not fatigue symptoms on a momentary basis (McLean et al, 2005b), also support the hypothesis that HPA axis hyperactivity is linked specifically to FM pain.

The relationship between CSF CRF levels and the activity of CRF-secreting neurons in the hypothalamic paraventricular nucleus (which influence pituitary-adrenal function) is not well understood. Evidence indicates that the great majority of CSF CRF originates from outside the hypothalamus (Garrick et al, 1987; Kalin et al, 1987; Kling et al, 1994), and CSF CRF levels do not correlate well with adrenocorticotropin and cortisol levels on a momentary basis (Geracioti et al, 1997). However, in rodent models lesioning the paraventricular nucleus decreases CSF CRF by 50-60\% (Hong et al, 1995; Gold and Chrousos, 2002). In humans, elevated CSF CRF levels have been found to be associated with a blunted adrenocorticotropin response to exogenously administered CRF (Newport et al, 2003). This blunted adrenocorticotropin response is believed to be due to chronic hyperactivity of CRF-secreting paraventricular neurons, suggesting that CSF CRF may be associated with paraventricular nucleus CRF secretion over the long term (Newport et al, 2003).

FM women in our sample who reported a history of physical or sexual abuse had significantly lower levels of CSF CRF than those who did not report such a history. This finding is consistent with that of Carpenter et al (2004), who found that perceived life stress during the preteen years was associated with decreased CSF CRF. The influence of selfreported childhood abuse has only recently been assessed in studies of stress axis function in FM (McLean et al, 2005b; Weissbecker et al, 2005), despite the fact that FM patients are often recruited for research studies from tertiary care clinic populations, where the prevalence of self-reported abuse is often $50 \%$ or more (Taylor et al, 1995; Goldberg et al, 1999; Carpenter et al, 2004). These recent studies describe relatively low cortisol levels in FM patients reporting a history of childhood abuse, and relatively high levels in those not reporting a history (McLean et al, 2005b; Weissbecker et al, 2005). These data and the present study suggest that FM patients with a history of abuse may have relatively low levels of basal CSF CRF and cortisol secretion, whereas those without such a history may have relatively high levels.

Some of the inconsistencies of the HPA literature regarding FM may be due to the failure to adjust for the confounding influence of abuse and other forms of early life stress. The results of this study suggest that important subgroups of FM patients may exist depending on early life stress exposure. Such neurobiological differences may also influence treatment response, thus early life stress exposure may be an important domain to include in both FM assessment and intervention studies.

The results of this study also suggest that the presence and severity of chronic pain may be another important domain to assess in CRF studies of other stress-related disorders such as depression and PTSD. The relationship between pain and depression is complex (Campbell et al, 2003). An average of $65 \%$ of patients with major depression have some degree of comorbid pain symptoms (Bair et al, 2003). Similarly, $20-30 \%$ of outpatient civilian samples with 
PTSD (Hubbard et al, 1995; Amir et al, 1997; Beckham et al, 1997 ) and $80 \%$ of combat veterans with PTSD (Beckham et al, 1997) have comorbid chronic pain. If there is also an association between pain and CSF CRF in these populations, this could account for some of the previously inconsistent results of studies examining CSF CRF concentration in these disorders.

The purpose of this study was to compare FM patient symptoms and CSF CRF levels, no matched control group was employed. Previous studies utilizing control groups have reported mean CSF CRF levels of $22-25 \mathrm{pg} / \mathrm{ml}$ among controls (Bremner et al, 1997; Carpenter et al, 2004; Williams et al, 2004). In the present study, men and women with FM not reporting an abuse history had CSF CRF levels substantially higher than this $(38.9$ and $35.0 \mathrm{pg} / \mathrm{ml}$, respectively), suggesting that FM in patients without a history of significant early life stress may be characterized by elevated CSF CRF levels.

This study has a number of limitations that should be considered when interpreting the results. First, the fact that our FM patients were recruited at a tertiary referral center, consented to a GCRC protocol involving a lumbar puncture, and were reimbursed for their participation suggests that they may not be typical of the FM population in the community. In addition, study participants were asked if they had ever been physically or sexually abused, but were not asked behaviorally specific questions (eg 'did anyone ever touch your genitals when you didn't want them to'). The definition of abuse in this study was thus not specifically defined, and relied solely on participants' own label of their past experience. This kind of 'label' only abuse assessment is a relatively insensitive method of identifying abuse victims compared with asking behaviorally specific questions, because a significant number of victims do not label their experiences as 'abuse' (Hamby and Gray-Little, 2000; Fricker et al, 2003). Thus, our method of identifying abuse history may not have identified all participants with an abuse history in the study. Error in classification would underestimate true differences between those with or without self-reported abuse, and could increase the inaccuracy of regression model estimates. However, the self-reported abuse prevalence among FM patients in our sample is similar to that reported in other FM tertiary care populations (Taylor et al, 1995; Goldberg et al, 1999; Carpenter et al, 2004), and the validity of the brief selfreport measure used in the study is supported by the lower CSF CRF levels found among those reporting abuse, which is consistent with the known biological affects of early life stress (Carpenter et al, 2004; Heim et al, 2004).

Results of this study indicate that current pain symptoms are associated with CSF CRF concentration among patients with FM. These data support the hypothesis that abnormalities in the 'stress response' are associated with pain symptoms in FM, and that this association is not explained by the known associations between stress response function and depressive symptoms. Early life stress also appears to influence adult CSF CRF levels; how the association between and CSF CRF level and FM pain symptoms is influenced by early life stress is not known. Further studies examining the relationship between CSF CRF and pain symptoms in FM and in other chronic pain and psychiatric disorders are needed, to confirm this finding and to determine the mechanisms involved in the association between CRF and pain.

\section{ACKNOWLEDGEMENTS}

Funding for this study came from the Department of the Army Grant DAMD-17002-0018 and Grant M01 RR-13297 from the National Center for Research Resources, National Institutes of Health. Dr McLean is funded by NIH K12 RR017607-01, and Dr Harris is funded by NIH/NCCAM K01 AT01111-01.

\section{REFERENCES}

Akselrod S, Gordon D, Ubel FA, Shannon DC, Berger AC, Cohen RJ (1981). Power spectrum analysis of heart rate fluctuation: a quantitative probe of beat-to-beat cardiovascular control. Science 213: 220-222.

Amir M, Kaplan Z, Neumann L, Sharabani R, Shani N, Buskila D (1997). Posttraumatic stress disorder, tenderness and fibromyalgia. J Psychosom Res 42: 607-613.

Arlt J, Jahn H, Kellner M, Strohle A, Yassouridis A, Wiedemann K (2003). Modulation of sympathetic activity by corticotropinreleasing hormone and atrial natriuretic peptide. Neuropeptides 37: $362-368$.

Bair MJ, Robinson RL, Katon W, Kroenke K (2003). Depression and pain comorbidity: a literature review. Arch Intern Med 163: 2433-2445.

Beckham JC, Crawford AL, Feldman ME, Kirby AC, Hertzberg MA, Davidson JR et al (1997). Chronic posttraumatic stress disorder and chronic pain in Vietnam combat veterans. J Psychosom Res 43: $379-389$.

Bremner JD, Licinio J, Darnell A, Krystal JH, Owens MJ, Southwick SM et al (1997). Elevated CSF corticotropin-releasing factor concentrations in posttraumatic stress disorder. Am J Psychiatry 154: 624-629.

Campbell LC, Clauw DJ, Keefe FJ (2003). Persistent pain and depression: a biopsychosocial perspective. Biol Psychiatry 54: 399-409.

Carpenter LL, Tyrka AR, McDougle CJ, Malison RT, Owens MJ, Nemeroff CB et al (2004). Cerebrospinal fluid corticotropinreleasing factor and perceived early-life stress in depressed patients and healthy control subjects. Neuropsychopharmacology 29: 777-784.

Catley D, Kaell AT, Kirschbaum C, Stone AA (2000). A naturalistic evaluation of cortisol secretion in persons with fibromyalgia and rheumatoid arthritis. Arthritis Care Res 13: 51-61.

Chrousos G, Gold P (1992). The concepts of stress and stress system disorders. Overview of physical and behavioral homeostasis. J Am Med Assoc 267: 1244-1252.

Clauw DJ, Chrousos GP (1997). Chronic pain and fatigue syndromes: overlapping clinical and neuroendocrine features and potential pathogenic mechanisms. Neuroimmunomodulation 4: 134-153.

Cook DB, Lange G, Ciccone DS, Liu WC, Steffener J, Natelson BH (2004). Functional imaging of pain in patients with primary fibromyalgia. J Rheumatol 31: 364-378.

Crofford L, Demitrack M (1996). Evidence that abnormalities of central neurohormonal systems are key to understanding fibromyalgia and chronic fatigue syndrome. Rheum Dis Clin N Am 22: 267-284.

Crofford LJ, Pillemer SR, Kalogeras KT, Cash JM, Michelson D, Kling MA et al (1994). Hypothalamic-pituitary-adrenal axis perturbations in patients with fibromyalgia. Arthritis Rheum 37: $1583-1592$. 
Fricker AE, Smith DW, Davis JL, Hanson RF (2003). Effects of context and question type on endorsement of childhood sexual abuse. J Trauma Stress 16: 265-268.

Fukuda K, Straus SE, Hickie I, Sharpe MC, Dobbins JG, Komaroff A (1994). The chronic fatigue syndrome: a comprehensive approach to its definition and study. International Chronic Fatigue Syndrome Study Group. Ann Intern Med 121: 953-959.

Garrick NA, Hill JL, Szele FG, Tomai TP, Gold PW, Murphy DL (1987). Corticotropin-releasing factor: a marked circadian rhythm in primate cerebrospinal fluid peaks in the evening and is inversely related to the cortisol circadian rhythm. Endocrinology 121: 1329-1334.

Geisser ME, Roth RS, Robinson ME (1997). Assessing depression among persons with chronic pain using the Center for Epidemiological Studies-Depression Scale and the Beck Depression Inventory: a comparative analysis. Clin J Pain 13: 163-170.

Geracioti Jr TD, Loosen PT, Orth DN (1997). Low cerebrospinal fluid corticotropin-releasing hormone concentrations in eucortisolemic depression. Biol Psychiatry 42: 165-174.

Gold PW, Chrousos GP (2002). Organization of the stress system and its dysregulation in melancholic and atypical depression: high vs low CRH/NE states. Mol Psychiatry 7: 254-275.

Goldberg RT, Pachas WN, Keith D (1999). Relationship between traumatic events in childhood and chronic pain. Disabil Rehabil 21: $23-30$.

Gracely RH, Petzke F, Wolf JM, Clauw DJ (2002). Functional magnetic resonance imaging evidence of augmented pain processing in fibromyalgia. Arthritis Rheum 46: 1333-1343.

Griep EN, Boersma JW, de Kloet ER (1993). Altered reactivity of the hypothalamic-pituitary-adrenal axis in the primary fibromyalgia syndrome. J Rheumatol 20: 469-474.

Hamby SL, Gray-Little B (2000). Labeling partner violence: when do victims differentiate among acts? Violence Vict 15: 173-186.

Heim C, Plotsky PM, Nemeroff CB (2004). Importance of studying the contributions of early adverse experience to neurobiological findings in depression. Neuropsychopharmacology 29: 641-648.

Hong SK, Gold PW, Herkenham M (1995). Hypothalamic paraventricular nucleus lesions decrease corticotropin releasing hormone in the CSF and elevate TH in mRNA in the locus ceruleus. Soc Neurosci Abstr 19: 762.

Hubbard J, Realmuto GM, Northwood AK, Masten AS (1995). Comorbidity of psychiatric diagnoses with posttraumatic stress disorder in survivors of childhood trauma. J Am Acad Child Adolesc Psychiatry 34: 1167-1173.

Kalin NH, Shelton SE, Barksdale CM, Brownfield MS (1987). A diurnal rhythm in cerebrospinal fluid corticotrophin-releasing hormone different from the rhythm of pituitary-adrenal activity. Brain Res 426: 385-391.

Kling MA, DeBellis MD, O’Rourke DK, Listwak SJ, Geracioti Jr TD, McCutcheon IE et al (1994). Diurnal variation of cerebrospinal fluid immunoreactive corticotropin-releasing hormone levels in healthy volunteers. J Clin Endocrinol Metab 79: 233-239.

Lariviere WR, Melzack R (2000). The role of corticotropinreleasing factor in pain and analgesia. Pain 84: 1-12.

Martinez-Lavin M, Hermosillo AG, Rosas M, Soto ME (1998). Circadian studies of autonomic nervous balance in patients with fibromyalgia: a heart rate variability analysis. Arthritis Rheum 41: 1966-1971.

McCain GA, Tilbe KS (1989). Diurnal hormone variation in fibromyalgia syndrome: a comparison with rheumatoid arthritis. J Rheumatol Suppl 19: 154-157.

McLean SA, Clauw DJ, Abelson JL, Liberzon I (2005a). The development of persistent pain and psychological morbidity after motor vehicle collision: integrating the potential role of stress response systems into a biopsychosocial model. Psychosom Med 67: 783-790.

McLean SA, Williams DA, Harris RE, Kop WJ, Groner KH, Ambrose $\mathrm{K}$ et al (2005b). Momentary relationship between cortisol secretion and symptoms in patients with fibromyalgia. Arthritis Rheum 52: 3660-3669.

Melzack R (1987). The short-form McGill Pain Questionnaire. Pain 30: 191-197.

Newport DJ, Heim C, Owens MJ, Ritchie JC, Ramsey CH, Bonsall R et al (2003). Cerebrospinal fluid corticotropin-releasing factor (CRF) and vasopressin concentrations predict pituitary response in the CRF stimulation test: a multiple regression analysis. Neuropsychopharmacology 28: 569-576.

Palkovits M (1999). Interconnections between the neuroendocrine hypothalamus and the central autonomic system. Geoffrey Harris Memorial Lecture, Kitakyushu, Japan, October 1998. Front Neuroendocrinol 20: 270-295.

Radloff LS (1977). The CES-D Scale: a self-report depression scale for research in the general population. Appl Psychol Meas 1: 385-401.

Radulovic D, Barbey JT, Katz P, Baraniuk J, Clauw DJ (1995). Tilt table testing as a measure of dysautonomia in fibromyalgia (FM). Arthritis Rheum 38: R25.

Riedel W, Layka H, Neeck G (1998). Secretory pattern of GH, TSH, thyroid hormones, ACTH, cortisol, FSH, and $\mathrm{LH}$ in patients with fibromyalgia syndrome following systemic injection of the relevant hypothalamic-releasing hormones. $Z$ Rheumatol 57: 81-87.

Riedel W, Schlapp U, Leck S, Netter P, Neeck G (2002). Blunted ACTH and cortisol responses to systemic injection of corticotropin-releasing hormone $(\mathrm{CRH})$ in fibromyalgia: role of somatostatin and CRH-binding protein. Ann NY Acad Sci 966: 483-490.

Smets EM, Garssen B, Bonke B, De Haes JC (1995). The Multidimensional Fatigue Inventory (MFI) psychometric qualities of an instrument to assess fatigue. J Psychosom Res 39: 315-325.

Stein PK, Domitrovich PP, Ambrose K, Lyden A, Fine M, Gracely $\mathrm{RH}$ et al (2004). Sex effects on heart rate variability in fibromyalgia and Gulf War illness. Arthritis Rheum 51: 700-708.

Stein PK, Kleiger RE (1999). Insights from the study of heart rate variability. Annu Rev Med 50: 249-261.

Taylor JA, Carr DL, Myers CW, Eckberg DL (1998). Mechanisms underlying very-low-frequency RR-interval oscillations in humans. Circulation 98: 547-555.

Taylor ML, Trotter DR, Csuka ME (1995). The prevalence of sexual abuse in women with fibromyalgia. Arthritis Rheum 38: 229-234.

Weissbecker I, Floyd A, Dedert E, Salmon P, Sephton S (2005). Childhood trauma and diurnal cortisol disruption in fibromyalgia syndrome. Psychoneuroendocrinology 31: 312-324.

Williams DA, Baraniuk J, Gracely RH, Whalen G, Lyden A, Clauw DJ (2004). Resting cerebrospinal fluid CRH levels differ between fibromyalgia and related conditions, and healthy controls. Arthritis Rheum 50: S249.

Wittchen HU (1994). Reliability and validity studies of the WHO-Composite International Diagnostic Interview (CIDI): a critical review. J Psychiatr Res 28: 57-84.

Wolfe F, Smythe H, Yunus M, Bennett R, Bombardier C, Goldenberg D (1990). The American College of Rheumatology 1990 Criteria for the Classification of Fibromyalgia. Report of the Multicenter Criteria Committee. Arthritis Rheum 33: $160-172$. 